\title{
Nosologie Des Enfants Dits Atypiques Dans La Societe Baoule (Cote D'ivoire)
}

\author{
KACOU Fato Patrice, \\ Docteur en socio-anthropologie, Chercheur à l'Institut d'Ethno-Sociologie de \\ l'Université Félix Houphouët Boigny d'Abidjan-Cocody (Côte d'Ivoire) \\ DOUKOURE DaOuda,
} Docteur en socio-anthropologie, Chercheur à l'Institut National de Santé Publique d'Abidjan (INSP) (Côte d'Ivoire)

ATSE Achi Amédée-Pierre, Docteur en sociologie, Département de Sociologie et anthropologie, Université Péléforo Gon Coulibaly de Korhogo (Côte d'Ivoire)

doi: 10.19044/esj.2016.v12n26p135 URL:http://dx.doi.org/10.19044/esj.2016.v12n26p135

\begin{abstract}
The study analyses the social mechanisms that govern the acceptance of children in Baoule society. It is clear to open a window on the social frames of reference from which, on the one hand, we refer to the so-called atypical children and on the other hand to classify them.. To do this, using qualitative approches, we collected data from a sexagenarian Baoulé tradi-prationer based in Bouaké, selected according to our target. The investigations led to the result that the social representations of pregnancy and child in the Baoulé society call for the observation of taboos during pregnancy and structure the typology of so-called mis fortune children. They also determine the social solutions to the cases of evil called children. That requires the urgent adoption of an interdisciplinary approch that puts together at least human and health sciences in the treatement of problems linked to infant mortality and beloyond, changes social perceptions in order to keep alive the so-called atypical children, codemned to death or socially marginalized.
\end{abstract}

Keywords: Baoulé, defformity, child, pregnancy, taboo

\section{Resume}

L'étude analyse les mécanismes sociaux qui président à l'acceptation des enfants dans la société Baoulé. Il s'agit en clair d'ouvrir une lucarne sur les cadres de références sociales à partir desquels d'une part l'on désigne les enfants dits atypiques et d'autre part les classifie. Pour ce faire, à l'aide d'approches qualitatives, les données ont été recueillies auprès d'une tradi- 
praticienne Baoulé, sexagénaire, et exerçant à Bouaké, sélectionnée par choix raisonné. Les investigations ont abouti au résultat que les représentations sociales de la grossesse et de l'enfant dans la société Baoulé appellent à l'observance de tabous en période de maternité et structurent la typologie des enfants dits de malheur. Elles déterminent également les réponses sociales à apporter aux cas des enfants dits maléfiques. Toute chose qui requiert l'urgence de l'adoption d'une approche interdisciplinaire réunissant au moins les sciences humaines et les sciences de la santé dans le traitement des problématiques sur la mortalité infantile et au- delà, modifier les perceptions sociales dans le sens de maintenir en vie les enfants dits atypiques, condamnés à mort ou à la marginalité sociale.

Mots Cles: Baoulé, difformité, enfant, grossesse, tabou

\section{Introduction}

En Côte d'Ivoire, chez le groupe ethnoculturel Baoulé, un système ethno-médical est bâti spécifiquement autour de la femme enceinte et de son enfant pour repousser le plus loin possible les limites de la maladie (handicap) et même de la mort. Ainsi, dès le début de la grossesse, la future parturiente est prise en charge sur plusieurs dimensions. Au plan social, la future parturiente est déchargée de toutes sortes d'activités ardues. De cette façon, elle suit médicalement un traitement consistant à badigeonner le ventre de kaolin et à boire des solutions médicamenteuses. Elle offre aussi des aliments en sacrifice (ATSE, 2013), en termes de prière, espérant que les acteurs symboliques (génies, esprits, etc.) lui donneront un enfant sociable (ATSE, 2015) et assureront sa protection. Du point de vue culturel, elle reçoit régulièrement des conseils et informations concernant ce qu'elle doit observer, surtout ce qu'elle doit ingurgiter. Et cela est transmis de génération en génération. Il existe donc, comme chez tous les peuples, un système classificatoire du mangeable et de l'immangeable (POULAIN, 2002). Cet appareil de catégories et de règles alimentaires (FISCHLER, 1990) renvoie à des logiques, des rationalités qui s'enracinent dans les représentations, l'imaginaire de la culture concernée (POULAIN, 2002). Manger donc chez les femmes enceintes en pays Baoulé s'inscrit dans un système de valeur et s'articule autour des logiques totémiques (LEVI-STRAUSS, 1962: 2), sacrificielles (DETIENNE et VERNANT, 1979) et hygiéniques (ATSE, 2013). Tout ce processus normatif qui encadre la gestion de la grossesse vise un accouchement normal, un développement psychomoteur harmonieux chez le fœtus et donc une réduction de la morbidité et de la mortalité néo-fœtales. Cela suppose que ce protocole permettrait à la société Baoulé d'éviter, les pathologies et malformations intra-utérines comme le mongolisme, la trisomie 21 , etc. Cependant, cette gestion exclusivement fondée sur la «pensée 
magique »(FISCHLER, 1994), sur des mythes et sur des idéologies doit collaborer avec la médecine moderne. En effet, malgré la ritualisation des pratiques sociales niant ces « accidents biologiques » par l'exorcisme, la société Baoulé compte toujours, des mongoliens, des albinos, toutes sortes de cas non souhaités, des décès en couches, etc. Pour preuve, sur la période 20072012, l'ensemble des régions de la Côte d'Ivoire a enregistré en moyenne des taux de $38 \%$ pour la mortalité néonatale et 30\%o pour la mortalité postnéonatale et le risque de mortalité est porté à 68\% (EDS-III, 2012). Pour la région du centre, concernée par notre étude, on enregistre des taux de $52 \%$ o pour la mortalité néonatale, $35 \%$ pour mortalité post-néonatale et $87 \%$ pour la mortalité infantile, (EDS-III, 2012 : 129). Ces taux dépassent de loin une principauté comme Monaco qui a un taux de mortalité infantile de $1,79 \%{ }^{11}$.

Au regard de ces données inquiétantes, l'Etat ivoirien et ses partenaires $\left(\mathrm{OMS}^{12}, \mathrm{UNICEF}^{13}\right.$, les $\mathrm{ONG}^{14}$, etc.) se sont engagés dans la promotion de la protection infantile et maternelle en construisant des maternités de proximité, en augmentant le personnel de santé (GOUBO, 2013), en distribuant gratuitement des moustiquaires imprégnées aux femmes enceintes et en vaccinant gratuitement les enfants de moins de cinq ans. Malgré ces efforts du gouvernement ivoirien, les Baoulés continuent de développer des mécanismes spécifiques de type médico-magiques ou magico-religieux de protection maternelle, néonatale et infantile. Cet ordre culturel qui fonde sa légitimité sur des croyances, des idéologies et des mythes alimentaires pourrait avoir des conséquences nutritionnelles, telle que la malnutrition. En effet, la privation ou les tabous temporaires de certains nutriments chez la gestante peut entraîner des troubles nutritionnels chez l'enfant et sa mère (DUPIN et RAIMBAULT, 1978).

Au regard de ce qui précède, il importe de mener des investigations dans le but de mettre en relief les représentations sociales de la grossesse et de l'enfant dans les sociétés de type traditionnel à l'image de celle des Baoulé. Ces investigations pourront, par exemple, permettre de comprendre certaines logiques sociales, imaginaires et culturelles susceptibles de bloquer des politiques de prévention, en matière de santé maternelle et infantile.

\section{Methodologie}

L'étude s'inscrit dans une approche socio-anthropologique qui consiste à faire ressortir les logiques représentationnelles d'une certaine catégorie d'enfants dans la société Baoulé et à se référer aux paradigmes de

\footnotetext{
${ }^{11}$ http://www.statistiques-mondiales.com/mortalite_infantile.htm, site consulté le 28 novembre 2013

${ }^{12}$ Organisation Mondiale de la Santé

${ }^{13}$ United Nations International Children's Emergency Fund

${ }^{14}$ Organisation Non Gouvernementale
} 
l'anthropologie des systèmes sanitaires. Les données ont été recueillies auprès d'une tradi-praticienne sexagénaire, issue de l'ethnie Baoulé et exerçant à Bouaké $^{15}$. Sa sélection a été guidée par la technique du choix raisonné. Nous avons eu avec elle, en deux jours d'entretien individuel approfondi, des données sur les représentations sociales de la grossesse et de l'enfant, les pathologies materno-infantiles, la nosographie des bébés ou enfants dits de malheur et les réponses apportées à ces types d'enfants. Au- delà des deux jours francs, nous sommes revenus à la tradi-praticienne pour des informations complémentaires. Cependant, il importe d'indiquer qu'on n'a pas eu accès à certaines informations soumises à la loi de l'omerta. Elles concernent notamment, les pratiques ou incantations susceptibles de mettre fin à la vie des enfants perçues indésirables.

Les données ont été enregistrées et transcrites manuellement et leur exploitation s'est appuyée sur la méthode de l'analyse de contenu. Ainsi, une liste de codes a-t-elle été dressée à partir du contenu des entretiens. Ces codes ont ensuite été insérés progressivement dans chaque entretien. C'est après cette démarche que les catégories d'idées ont été regroupées et que l'analyse finale s'est faite en fonction de chaque catégorie. Des verbatim ont été sélectionnés pour illustrer chaque catégorie d'idées faisant l'objet du présent article.

Du dépouillement des données, on note quatre résultats essentiels: les représentations sociales de la grossesse et de l'enfant dans la société Baoulé, les tabous en période de maternité, la typologie des enfants dits de malheur 'tété-ba', les réponses sociales aux enfants dits maléfiques.

\section{Resultats}

\section{Les representations sociales de la grossesse et de l'enfant dans la societe baoule}

Le peuple Baoulé, champ social de cette étude, doit son appellation ethnique 'Baoulé" à la légende de la Reine Abla Pokou. Baoulé est la déformation de la prononciation canonique de 'baouli' qui signifie l'enfant est mort. Selon cette légende, l'actuel peuple Baoulé a fui le conflit de succession qui a éclaté suite à la mort de Osei Tutu, Roi des Ashanti vers le XVIII ${ }^{\text {ème }}$ siècle au Ghana, pour s'installer au Centre de la Côte d'Ivoire actuelle. Lors de l'exode, le peuple était confronté à la difficile traversée d'un cours d'eau. Pour y parvenir, le génie des eaux a demandé un sacrifice humain. C'est alors que Abla Pokou, la sœur du Chef qui menait l'exode, s'est résolue à sacrifier son fils, neveu du chef, et dauphin au trône ${ }^{16}$.

\footnotetext{
${ }^{15}$ Bouaké: deuxième grande ville après Abidjan, située au Centre de la Côte d'Ivoire.

${ }^{16}$ On est dans une société dite matriarcale.
} 
Dans la société Baoulé en général, la procréation ' 'baoulè" n'est pas seulement un processus biologique, elle est plus métaphysique. C'est un don que les entités surnaturelles accordent aux familles; c'est le fruit du mariage, l'élément structurant de l'alliance entre les familles. Comme tel, des prières (libation: 'n'zan asségoualè', sacrifice ' 'tê"') sont dites pour que la grossesse "kouè" se déroule sans heurt. Des conditions déviantes dans l'état de grossesse conduisent à supprimer la vie de bébés (prise de grossesse avant le rite consacrant la puberté "attovlè"). L'anthroponomie ou l'attribution de noms aux nouveau-nés peut être fonction du jour de naissance, du nom du génie bienfaiteur ou des circonstances de la période de maternité. En outre, ne pas être féconde amène à interroger les oracles ou médiums 'komian", courroies de transmission entre le monde visible et les dieux, les génies ou ancêtres, pour en savoir la cause. La stérilité peut être due à l'adultère, à la transgression des interdits, à l'envoûtement, à la sorcellerie, à la malédiction, à des conflits familiaux. C'est la raison pour laquelle, dès la conception de l'enfant et dans son évolution, on demande la bienveillance des entités surnaturelles à son égard. On parle chez les Baoulé de mari de l'au-delà 'blolo-bian"' et de femme de l'au-delà 'blolo-bla' qui peuvent, en cas de mécontentement, empêcher la procréation dans un couple, donner des enfants anormaux du point de vue physiologique, ou introduire le désordre dans le déroulement de sa trajectoire de vie sociale. Par extrapolation, on peut citer les mythologies égyptienne et grecque qui désignent respectivement les dieux de la fertilité et de la fécondité sous les noms de Min et Priape.

Au nom de cette conception, il est attaché une déférence particulière à la femme enceinte. Elle jouit d'une protection voire d'une immunité sociale en cas de conflit. On ne s'en prend pas à une femme enceinte. La femme enceinte est soumise à l'observance de règles et pratiques (confère tableau $\mathrm{n}^{\circ} 1$ ). Ce rapport au monde profite également aux animaux en gestation. Par ces mesures, on fabrique des idéologies dont les postulats implicites fonctionnent comme un mode de pérennisation de l'espèce humaine et de la faune. C'est ainsi qu'il est formellement interdit d'abattre un animal en gestation sous peine d'être frappé pas la colère des génies ou de rencontrer le malheur dans sa trajectoire de vie. On croit que l'enfant comprend le langage des animaux; il est aussi capable de comprendre le monde invisible. On va jusqu'à concevoir qu'il ne lui arriverait rien ou qu'il serait épargné des malheurs et accidents du fait de sa proximité avec les entités surnaturelles. C'est ce qu'on convient d'appeler le mythe de l'enfant innocent. Si l'on considère l'enfant comme un élu des dieux, il n'en est pas de même pour la mère qui est soumise pendant et après la maternité à des interdits, dominés par les totems et tabous alimentaires. 


\section{Les tabous en periode de maternite}

Chez les Baoulé, la période prénatale est délicate et donc vécue avec précaution. Pour ce faire, la femme enceinte " kouè-fouè" est astreinte à un certain nombre d'interdits à observer sous peine de compromettre le développement normal de la grossesse ou de mettre au monde un bébé atypique "tété-ba". Ces interdits 'tchiliè" prennent sources dans les superstitions du milieu symbolisées par des éléments porteurs de négativité tels que la nuit " kongouè", les pleurs " sounlè", les larmes " imouin-zué" et la mort "vié". Ainsi, il est défendu à une femme enceinte de prendre son bain nuitamment, de manger avant de prendre son bain le matin, de voir un animal mort, de voir un caméléon vivant ou mort, de pleurer jusqu'à laisser tomber ses larmes sur le ventre, et d'entendre parler de morts ou de choses néfastes. La transgression des interdits sus évoqués entrainerait des conséquences. Par souci de compréhension, on les range dans le tableau cidessous.

Tableau $\mathbf{n}^{\circ}$ 1: observance de pratiques sociales chez la femme en période de maternité

\begin{tabular}{|c|c|c|c|c|}
\hline $\mathrm{N}^{\circ}$ & Interdits & $\begin{array}{c}\text { Idéologies liées aux } \\
\text { interdits }\end{array}$ & $\begin{array}{l}\text { Exorcisme de } \\
\text { menaces }\end{array}$ & $\begin{array}{l}\text { Objectifs } \\
\text { implicites } \\
\text { liés aux } \\
\text { idéologies }\end{array}$ \\
\hline & $\begin{array}{l}\text { Ne pas prendre } \\
\text { son bain la nuit. }\end{array}$ & $\begin{array}{l}\text { Les génies se lavent la } \\
\text { nuit. Ils échangent les } \\
\text { enfants avec les siens. Et } \\
\text { les enfants peuvent naître } \\
\text { malformés. }\end{array}$ & $\begin{array}{l}\text { Mise à mort de } \\
\text { l'enfant né avec des } \\
\text { malformations. }\end{array}$ & $\begin{array}{l}\text { La gestante } \\
\text { peut glisser } \\
\text { la nuit et } \\
\text { tomber sur } \\
\text { son ventre à } \\
\text { cause de } \\
\text { l'absence de } \\
\text { l'électricité } \\
\text { dans les } \\
\text { milieux } \\
\text { traditionnels. }\end{array}$ \\
\hline & $\begin{array}{c}\text { Ne pas manger } \\
\text { le matin avant } \\
\text { de prendre son } \\
\text { bain. }\end{array}$ & $\begin{array}{l}\text { Risque de paresse chez la } \\
\text { mère. }\end{array}$ & $\begin{array}{l}\text { Il n'existe que la } \\
\text { prévention qui } \\
\text { consiste à observer } \\
\text { l'interdit. }\end{array}$ & $\begin{array}{l}\text { Sous la faim, } \\
\text { donc faible, } \\
\text { elle peut } \\
\text { également } \\
\text { tomber. }\end{array}$ \\
\hline & $\begin{array}{c}\text { Ne pas voir les } \\
\text { cadavres } \\
\text { d'animaux. }\end{array}$ & $\begin{array}{c}\text { Risque de mettre au } \\
\text { monde un enfant malade } \\
\text { dont la mort peut arriver } \\
\text { quelques jours après la } \\
\text { naissance. }\end{array}$ & $\begin{array}{l}\text { Couper des herbes } \\
\text { et les déposer sur } \\
\text { les cadavres } \\
\text { d'animaux. } \\
\text { Soumission du } \\
\text { nouveau-né à un } \\
\text { rituel expiatoire. }\end{array}$ & $\begin{array}{l}\text { Refus par } \\
\text { principe de } \\
\text { similitude, la } \\
\text { mort du } \\
\text { fœtus. }\end{array}$ \\
\hline & $\begin{array}{l}\text { Ne pas voir le } \\
\text { caméléon vivant } \\
\text { ou mort. }\end{array}$ & $\begin{array}{c}\text { Probable mort du } \\
\text { nouveau-né quelques } \\
\text { jours après sa naissance }\end{array}$ & $\begin{array}{l}\text { Se servir d'un fouet } \\
\text { ou bois pour } \\
\text { frapper le caméléon. }\end{array}$ & $\begin{array}{c}\text { Refus des } \\
\text { propriétés } \\
\text { symboliques } \\
\text { (lenteur, }\end{array}$ \\
\hline
\end{tabular}




\begin{tabular}{|c|c|c|c|}
\hline & $\begin{array}{l}\text { (la couleur de la peau du } \\
\text { bébé change à sa mort). }\end{array}$ & $\begin{array}{l}\text { Soumission de la } \\
\text { mère à un rituel } \\
\text { expiatoire. }\end{array}$ & $\begin{array}{l}\text { changement } \\
\text { ou caractère } \\
\text { versatile) du } \\
\text { caméléon. }\end{array}$ \\
\hline $\begin{array}{l}\text { Ne pas pleurer } \\
\text { jusqu'à laisser } \\
\text { tomber ses } \\
\text { larmes sur le } \\
\text { ventre. }\end{array}$ & $\begin{array}{c}\text { Risque de malédiction } \\
\text { sur la grossesse (mort } \\
\text { 'sipè,", accouchement } \\
\text { difficile). }\end{array}$ & $\begin{array}{l}\text { Il n'existe que la } \\
\text { prévention qui } \\
\text { consiste à observer } \\
\text { l'interdit. }\end{array}$ & $\begin{array}{l}\text { Refus d'un } \\
\text { enfant } \\
\text { pleurnichard } \\
\text { et possibilité } \\
\text { de tomber } \\
\text { malade en } \\
\text { pleurant. }\end{array}$ \\
\hline $\begin{array}{c}\text { Eviter } \\
\text { d'entendre } \\
\text { parler de morts } \\
\text { ou de choses } \\
\text { néfastes. }\end{array}$ & $\begin{array}{l}\text { Risque de malédiction } \\
\text { sur la grossesse. }\end{array}$ & $\begin{array}{l}\text { Cracher sur son } \\
\text { ventre. }\end{array}$ & $\begin{array}{l}\text { Refus } \\
\text { d'incorporer } \\
\text { ou d'être } \\
\text { hanté par la } \\
\text { mort chez la } \\
\text { gestante. }\end{array}$ \\
\hline $\begin{array}{l}\text { Ne pas outrager } \\
\text { ou mépriser les } \\
\text { enfants infirmes } \\
\text { ou difformes. }\end{array}$ & $\begin{array}{l}\text { Risque de survenue de la } \\
\text { même infirmité chez } \\
\text { l'enfant en conception. }\end{array}$ & $\begin{array}{l}\text { Il n'existe que la } \\
\text { prévention qui } \\
\text { consiste à observer } \\
\text { l'interdit. }\end{array}$ & $\begin{array}{c}\text { Risque } \\
\text { d'avoir un } \\
\text { enfant } \\
\text { infirme. }\end{array}$ \\
\hline $\begin{array}{l}\text { Ne pas vanter } \\
\text { l'enfant encore } \\
\text { en gestation au } \\
\text { détriment des } \\
\text { autres. } \\
\end{array}$ & $\begin{array}{l}\text { Susceptible de provoquer } \\
\text { la jalousie des sorciers } \\
\text { (provoque la mort). }\end{array}$ & $\begin{array}{l}\text { Il n'existe que la } \\
\text { prévention qui } \\
\text { consiste à observer } \\
\text { l'interdit. }\end{array}$ & $\begin{array}{c}\text { Eviter } \\
\text { l'attaque des } \\
\text { forces } \\
\text { maléfique. }\end{array}$ \\
\hline
\end{tabular}

L'ensemble de ces interdits visent à empêcher trois choses: la perte de la vie de l'enfant, la mise au monde d'un enfant difforme et un parcours de vie controversée à l'enfant (un enfant envieux, voleur).

En outre, plusieurs totems et tabous alimentaires de types temporaires sont observés chez la femme gestante. On note l'interdiction de la consommation de la chair de singe, du chat huant (woya), du cœur des animaux et des œufs. En cas de violation de ces interdits, la mère risque en plus des sanctions énumérées dans le tableau ci-dessus, une hémorragie à l'accouchement. A l'homme, auteur de la grossesse, il lui est recommandé d'éviter d'entrer en conflit avec sa femme, car l'enfant de sexe masculin peut trouver la mort (sipè). Cette idéologie vise à éloigner les enfants (généralement friands des œufs) des œufs garantissant ainsi la pérennité de la basse-cour et évitant des enfants voleurs. De façon analogue, les Baoulé refusent les propriétés symboliques de l'aliment consommé. Le chimpanzé ressemblant à l'homme, la femme enceinte refuse sa consommation pour deux raisons: elle ne veut pas etre cannibale et refuse que son enfant ressemble au chimpanzé. Ce même principe fonctionne avec le chat huant (woya). On ne veut pas que l'enfant pleure et crie comme cet animal. Ces croyances s'expliquent par trois principes, le principe d'incorporation (on devient ce qu'on mange, c'est-àdire on copie les propriétés symboliques de l'aliment ingéré), le principe de 
similitude (on ressemble à ce qu'on mange) et le principe de distanciation ou de proximité symbolique.

Mais en partant du postulat qu' « on devient ce qu'on mange », les Baoulé encourage la consommation de la viande de l'écureuil chez la femme enceinte. Ici, on recherche la rapidité de l'animal. On souhaiterait que l'accouchement soit facile et que l'enfant acquiert rapidement certaines capacités psychomotrices comme la marche. Cependant, le système de signification peut différer d'un peuple à l'autre. Ainsi, un déséquilibre dans les rapports entre la mère ou la famille et les normes sociales peut entraîner des types particuliers d'enfants.

\section{LA TYPOLOGIE DES ENFANTS DITS DE MALHEUR “ 'tété- $b a$ '”}

Dans la société Baoulé, on a deux grands types d'enfants. Les enfants nés normaux et les enfants nés avec des difformités. Les enfants nés normaux sont ceux considérés comme ayant la faveur des entités surnaturelles (dieux, génies, ancêtres). En effet, l'enfant d'une telle naissance est la preuve du déroulement de la grossesse en rapport avec les normes sociales, d'une famille harmonieuse. Les forces maléfiques (sorciers) ne s'attaquent qu'aux déviants. Les enfants nés avec des difformités sont ceux frappés de sanctions pour causes de transgression et de conflits intrafamiliaux. On a quatre sous-types d'enfants nés avec des difformités qui elles-mêmes sont fonction de leurs causes.

Tableau $\mathbf{n}^{\circ}$ 2: typologie des enfants dits atypiques ' 'tété-ba',

\begin{tabular}{|c|c|c|}
\hline $\mathrm{N}^{\circ}$ & Types d'enfants difformes & Causes des difformités \\
\hline wo $=$ serpent & $\begin{array}{c}\text { Si le génie de la termitière n'a pas agré le } \\
\text { sacrifice d'adoration accompli par les parents. }\end{array}$ \\
\hline terre & djué = poisson & $\begin{array}{c}\text { Si le génie de la forêt n'a pas agré le sacrifice } \\
\text { d'adoration accompli par les parents. }\end{array}$ \\
\hline folè = handicap & $\begin{array}{c}\text { Si le génie de l'eau n'a pas agréé le sacrifice } \\
\text { d'adoration fait par les parents. }\end{array}$ \\
\hline $\begin{array}{c}\text { Il y a un déséquilibre social qui cause le handicap } \\
\text { chez l'enfant. Ici, il s'agit d'une infirmité. } \\
\text { L'enfant ne marche pas, mais il peut se déplacer } \\
\text { en faisant les quatre pattes. Donc l'enfant est } \\
\text { maintenu en vie. }\end{array}$ \\
\hline
\end{tabular}

Dans le rapport au monde des Baoulé, les cours d'eau, la forêt, la termitière sont les réceptacles des forces surnaturelles (génies). Chaque groupe ou famille est affilié à un génie particulier dont elle connait les secrets d'adoration, les interdits et les vertus (bénédictions). Cependant, les rituels d'adoration, les conditions de culte peuvent ne pas être agréées par les génies parce que n'étant pas conformes à leurs prescriptions. Dans ce cas, le sacrifice "'tê"' est rejeté par les génies. Ainsi, selon l'entité spirituelle dont on se réclame, on peut mettre au monde un enfant anormal comme décrit dans le tableau $\mathrm{n}^{\circ} 2$ ci-dessus. Etant donné que l'enfant est considéré comme 
maléfique par la société, il y a des agents sociaux qui possèdent des secrets adaptés à la mise à mort de l'enfant dans la discrétion, à l'insu du commun des mortels.

Il y a aussi des enfants qui sont considérés comme maléfiques à cause de leur ordre de naissance chez la mère ou de l'inobservance de certains rituels avant la survenue de la grossesse. En effet, chez certains Baoulé, le douzième enfant appelé $n$ 'gbin, un enfant de sexe différent après la naissance de deux enfants du même sexe "kindo" est perçu comme maléfique. Il faut donc supprimer sa vie pour éviter des ennuis à son groupe social. C'est le sort similaire auquel on soumet l'enfant né avant que la mère n'accomplisse le rituel de la puberté (Wousilè). Ce rituel consiste, à asperger d'eau par une femme désignée par la famille, tôt le matin, pendant que la jeune fille est entrain de dormir. La femme dit cette phrase: «tes parents m'envoie te dire que tu as grandi ' wagni', (tu es devenue mature). Chez d'autres sous-groupes Baoulé, cette cérémonie a lieu juste après les premières menstrues chez la jeune fille pubère. Chez le groupe Baoulé-Agba par exemple, on lave à l'eau la jeune fille, on enduit son corps de beurre de karité ou de l'huile de palme, on recouvre son corps de kaolin blanc ( $n$ 'glo). On porte à la jeune fille un linge blanc et une ceinture aux cuisses. Les seins restent visibles, elle se pare de bijoux en perles. Elle fait ensuite une procession à travers les rues du village. On appelle la jeune fille "attovlè" pour traduire son aptitude au mariage.

C'est donc une infamie pour les familles de constater une grossesse avant ladite cérémonie. Pour réparer ce sacrilège la famille met à mort les enfants nés de pareilles grossesses. Ces types d'enfants sont appelés, 'abo n'gbinnouba". Le terme baoulé de "abo n'gbin-nouba" véhicule l'idée que la mère est une déviante sociale. Il faut mettre à mort l'enfant pour éviter que le comportement de la mère se reproduise chez d'autres demoiselles. Mais l'acte de mis à mort est souvent justifié par un test traditionnel (que seuls les initiés maîtrisent) qui révèle que l'enfant est un attardé psychique ou déformé qui ne mérite pas de vivre. De plus, le corps de l'enfant 'accompagné" doit être rapidement enterré à l'insu du public. Plusieurs réponses sont, de cette façon, socialement construites et adaptées à chaque type d'enfants dits maléfiques.

\section{LES REPONSES SOCIALES AUX ENFANTS DITS MALEFIQUES} 'tété-ba'"

Dans la société Baoulé, en dehors des enfants dits normaux donc ordinaires, il existe trois sous-types d'enfant de malheur. Ce sont les enfants conçus en l'absence de rituel de puberté, les enfants de malheur selon l'ordre de naissance et les enfants atypiques. 


\section{Les enfants conçus en l'absence de rituel de puberté "'Wousilè',}

Ici, l'enfant peut naître sans déformation. Cependant, on n'admet pas la mise au monde d'un enfant avant que la mère se soit soumise au rituel consacrant la puberté. Ce refus d'un tel enfant se manifeste par la suppression de sa vie selon des procédés secrets que connaissent des agents sociaux mis à part.

\section{Les enfants considérés maléfiques selon l’ordre de naissance}

Chez les Baoulé de même qu'il existe des jours perçus comme néfastes (mardi, mercredi, vendredi selon les différents groupes composant l'ethnie Baoulé), il existe un ordre de naissance dit maléfique. Il s'agit du douzième enfant ' 'n'gbin'”. Le terme Baoulé “'n'gbin' ' traduit la nullité, la futilité ou la vanité. L'enfant de cet ordre est mis à mort bien qu'il soit normal physiologiquement.

\section{Les enfants dits atypiques "'tété-ba',}

Les enfants atypiques sont ceux nés avec des difformités. Les difformités sont la preuve du malheur ou de la rupture du consensus avec les entités surnaturelles. Selon 1'entité spirituelle dont se réclament les parents ou familles, l'enfant atypique est appelé: '‘wo', ' 'assiè-oussou'; 'djué, ou 'folè'. Selon l'explication socioculturelle du milieu, l'enfant naît difforme parce que ses parents sont en rupture avec l'entité protectrice. Soit ils ont enfreint aux tabous, soit ils n'ont pas satisfait aux exigences des génies en termes de rituel d'adoration. Une fois l'enfant est conçu, il n'existe pas de remèdes pour réparer sa difformité. Les deux solutions qui s'offrent aux parents et familles sont d'abord la mise à mort de l'enfant et ensuite des sacrifices expiatoires au bénéfice des génies pour que cessent le malheur 'tété', et les sanctions. On ne peut pas cohabiter sous une forme visible avec les entités surnaturelles qui par essence sont des esprits. Ils appartiennent à l'au-delà ' 'blolo'. Ce serait la peur en permanence. On fait appel à des agents sociaux commis à ces fonctions qui immolent un animal (mouton, poulet etc.) ou prononcent des incantations à la suite desquelles meurt l'enfant. On n'emploie pas les thèmes de mourir ou de tuer. Par euphémisme, on parle d'accompagner l'enfant qui se traduit en Baoulé par ' 'yékô souman ba' (nous partons accompagner l'enfant). On accompagne quelqu'un pour qu'il retourne d'où il vient. C'est donc un étranger encombrant à qui on refuse l'hospitalité.

\section{Discussion}

La question des représentations sociales de la grossesse et de l'enfant pose la problématique de l'intégration au sein de la société d'une certaine catégorie de membres. Ces représentations commencent à se mettre en place 
dès la grossesse pour connaître leur dénouement à la naissance de l'enfant de ce qu'il peut vivre parce que sa naissance est conforme aux normes sociales ou il doit retourner (mourir) parce qu'il est source de malheur. Comme le dit ARIES ${ }^{17}$, dans chaque société, "il existe un type d'homme normal, et tout ce qui s'éloigne est plus ou moins pathologique...» (ARIES, 2003: 131-138). Avec FOUGEYROLLAS ${ }^{18}$, on voit que " cette normalité produit de la culture, règle donc l'intégration ou l'exclusion de certains individus en fonction de caractéristiques corporelles ou fonctionnelles.» (FOUGEYROLLAS, 1983).

De la grossesse en passant par l'enfantement, jusqu'aux maladies contractées soit par la mère soit par l'enfant, on explique tout en rapport avec les forces surnaturelles, des croyances parfois opposées à la biomédecine. De la sorte, "l'écureuil est prohibé aux femmes enceintes chez les Fang du Gabon parce que cet animal se réfugie dans les cavités des troncs d'arbre et la future mère, qui consommerait sa chair, risquerait que le foetus n'imite l'animal et refuse d'évacuer l'utérus.» (LEVI-STRAUSS, 1962: 1). Dans tous les quatre grands groupes socio-linguistiques (Akan, Gur, Krou, Mandé) que compte la Côte d'Ivoire, il y a la présence de l'idée d'enfants dits atypiques. Dans plusieurs cultures ivoiriennes, l'origine de l'existence humaine est attribuée à Dieu. Tout enfant porteur d'une différence (qu'elle soit organique ou psychique) est souvent perçu comme un enfant particulier, pas comme les autres. Cette perception de l'enfant singulier ou atypique, se retrouve dans les sociétés extra-occidentales (AYOSSO, $2005: 5)$. Ainsi, à titre d'illustration, chez les Sénoufo, les enfants atypiques se nomment ''logô", chez les Koulango, ' 'hiwo'. En extrapolant ces faits à ceux du Burkina Faso, on voit que chez les Mossi, les kinkirsi sont des puissances représentées sous forme de nain à grosse tête et longue chevelure habitant des lieux retirés de la brousse. Ils peuvent se lier à la destinée d'une personne (HOUIS, 1963: 120121). Par ailleurs, pour BONNET (1994), ces kinkirsi donnent naissance à des enfants kinkirga. Ce sont des enfants qui ont un comportement qui n'est pas normal ou qui sont nés avec une malformation. Ils n'ont la plupart du temps pas accès au langage. Parfois la naissance d'un tel enfant est attribuée à une faute commise par la mère. La relation est aussi établie entre l'attitude de la mère et le symptôme de l'enfant. Par ailleurs, la femme aurait dérangé un génie ou l'aurait piétiné. Pour la punir, il pénètre en elle à l'occasion d'un rapport sexuel conjugal et devient l'enfant dont elle accouchera.

Cependant, la médecine se démarque de cette conception des choses. Elle parle plutôt d'agénésie, de malformation fœtale, de souffrance fœtale ou

\footnotetext{
${ }^{17}$ Les attitudes devant les handicapés, la nouvelle revue de l'AIS, Centre National d'Etude et de formation pour l'enfance inadaptée, $n^{\circ} 23,2003$, pp 131-138)

${ }^{18}$ Entre peaux: logis de la différence du handicap à l'autonomie, mémoire de maîtrise, Québec, Université de Laval, 1983, p 1
} 
de déficit psychomoteur. Les causes de ces malformations sont en partie liées aux anomalies hématologiques, aux malformations génétiques et aux carences en acide folique. Les sociétés africaines, en particulier celles de la Côte d'ivoire, attribuent ces malformations souvent au non respect des tabous, surtout ceux en rapports avec l'alimentation, car celle-ci fait directement corps avec l'enfant et peut le modifier (POULAIN, 2002). Chez les Akyé ${ }^{19}$, la consommation des oufs est interdite aux enfants de moins de 5 ans sous prétexte que les œufs deviennent des vers dans le ventre des enfants (ATSE et ADON, 2015).

De même que les handicapés peuvent être intégrés dans la société en leur aménageant des espaces, de même les enfants nés avec des difformités peuvent remplir des rôles. Dans cet élan, HAMPATE-BA, puissant ses réflexions dans la sagesse peuhl, pose que "Toute difformité est signe de mystère, soit maléfique soit bénéfique" (HAMPATE-BA, 1978: 51). Bénéfique pour ceux qui savent s'en servir, ceux qui ont en eux des valeurs d'altruisme. Maléfiques pour ceux qui les relèguent et les rejettent.

Chez les Serer et les Lébou du Sénégal, le nit-ku-bu (ZEMPLENI et RABAIN, 1965) peut être reconnu comme tel après sa naissance du fait d'un cri particulier chez lui, son refus de téter, l'acquisition de la marche à sept mois. C'est un enfant bien bâti aux gros yeux et au teint clair. Il a une grossesse tête, ne regarde pas les gens en face, ne parle pas ou très peu. C'est un enfant qui manifeste des difficultés de communication, certaines anomalies du comportement et certaines particularités physiques. Le nit-ku-bu peut décider de sa propre mort. C'est un enfant fragile pouvant tomber malade à tout instant. Il est perçu à la fois comme pouvant apporter le bonheur (bénéfique) mais aussi le malheur (maléfique). C'est un étranger dont on n'est pas sûr de l'identité puisqu'il peut s'agir d'un ancêtre réincarné, ou d'un enfant de mère yaradal (mère ayant perdu plusieurs enfants). Il ne respecte ni les lois, ni les demandes de son entourage, il est « hors de l'ordre social symbolique, maître de ses actes, spectateur du monde des vivants qui n'a ni autorité ni pouvoir sur lui » (COLLOMB, 1985: 413).

Par ailleurs, dans certaines cultures, les jumeaux sont considérés comme des êtres mi-humains, mi-surhumains qui auraient des dons exceptionnels. Ils seraient conçus, l'un par un esprit et l'autre par un être normal (KOUASSI, 1997). La naissance des jumeaux peut générer des conduites tant positives que négatives. Chez les Dogon, les Bambara et les Malinké, les jumeaux rappellent et symbolisent un idéal mythique. (KOUASSI, op.cit.). En revanche, chez les Akikuya et les Akkansa d'Afrique Orientale, de même chez les Lubas (ex-Zaîre) et en Casamance, la naissance des jumeaux est signe de malheurs. (AYOSSO, $2005: 10$ ). Or, rien en soi n'est

\footnotetext{
${ }^{19}$ Groupe ethnique vivant majoritairement dans l'Est de la Côte d'Ivoire.
} 
maléfique à part ce que la société en rapport avec ses représentations a voulu que ce soit ainsi. Sous la pression des associations des droits humains, aujourd'hui on parle d'handisport, d'écriture braille. Ces mêmes handicapés, étaient autrefois frappés d'ostracisme. On a la légende de Soundjata (DJIBRIL, 1960) qui enseigne comment marginalisé en tant que handicapé, il a pu se hisser à la tête de l'empire Mandingue.

La société Baoulé nomme les enfants considérés comme atypiques à partir du principe de ressemblance (BIBEAU, 1978). Les comportements et les gestes de l'enfant sont renvoyés à ceux des autres êtres de la nature (animaux, poissons). D'où la catégorisation des enfants. Le terme d'accompagner utiliser pas souci d'euphémisme en lieu et place de mettre à mort est une stratégie de négation du crime, une opération psychologique pour atténuer en soi l'effet que peut susciter l'acte de mort au regard de la morale, c'est un lavage de cerveau. Des théories sont conçues pour modifier la conviction de ne pas tuer.

\section{Conclusion}

Cet article se positionne comme une contribution à la socioanthropologie des pratiques sociales en santé maternelle et infantile dans une société particulière de la Côte d'Ivoire, la société Baoulé. En effet, ce texte a mis en relief les présentations et les mécanismes sociaux à partir desquels on détermine l'essence d'un enfant en termes d'enfant normal ou atypique.

Les logiques sociales contrastent avec celles de la biomédecine dans la mesure où c'est dans le comportement des acteurs sociaux, leur relation avec l'environnement social (divinités, interdits etc.) que se trouvent les causes des malformations chez l'enfant. De façon générale, les réponses sociales commandent de mettre à mort l'enfant dit anormal (accompagner l'enfant). Cet ''accompagnement'" est souvent fait dans la discrétion. Les symboles utilisés pour les désigner font référence aux êtres vivants dans la forêt, l'eau ou aux génies. Ce qui suscite une attitude de peur.

$\mathrm{Au}$ moment où les Etats, notamment les Etats des pays économiquement faibles et les institutions internationales se mobilisent pour réduire le taux de mortalité infantile, il serait indiqué que les sciences sociales et les sciences de la santé s'accordent, au nom de l'interdisciplinarité, pour développer des approches qui intègrent ces types de pratiques dans le but de modifier les perceptions sociales dans le sens de maintenir en vie les enfants dits atypiques, condamnés à mort ou à la marginalité sociale.

\section{References:}

ARIES Philippe (2003), «Les attitudes devant les handicapés », in La nouvelle revue de l'AIS, Centre National d'Etude et de Formation pour l'enfance inadapté, $\mathrm{n}^{\circ}$ 23, pp. 131-138. 
ATSE Amédée-Pierre (2015), « De la perception de la malnutrition dans les localités rurales du département d'Adzopé », Revue Ivoirienne des Lettres, Arts et Sciences Humaines no 24 :59-72.

ATSE Amédée-Pierre, ADON Kouadio (2015), "Laisse-moi manger ta viande ». Le partage des protéines chez les Akyé, Journal des anthropologues, n¹40-141: 193-2012.

ATSE Amédée-Pierre (2013), Comportement alimentaire et santé en Côte d'Ivoire : le cas du District sanitaire d'Adzopé, Thèse unique de doctorat de sociologie. Abidjan, Université Félix Houphouët-Boigny.

AYOSSO Judith (2005), l'enfant tohossou: ses représentations dans la dynamique familiale chez les fronan Bénin, Master recherche, Université Paris 13, $93 \mathrm{p}$.

BIBEAU Gilles (1978), «L'organisation Ngbandi des noms de maladies », in Anthropologie et Sociétés, vol. 2, n 3, pp. 83-116.

BONNET Doris (1994), L'éternel retour ou le destin singulier, de l'enfant, in L'homme XXXIV (3), 93-110.

COLLOMB Henri (1985), Etudes transculturelles, in S. Lebovici, R. Diatkine, M. Soulé (EDS) nouveau traité de psychiatrie de l'enfant et de l'adolescent 1, 363-421 Paris PUF (Quadrige manuels).

CORBEAU Jean-Pierre; POULAIN Jean-Pierre (2002), Penser l'alimentation. Entre imaginaire et la rationalité, Toulouse, Privat, Ocha.210 pages.

Côte d'Ivoire, Ministère de la santé et de la lutte contre le sida. Institut National de la Statistique (2012). Enquête Démographique et de Santé et à indicateurs Multiples. EDSCI-III Rapport préliminaire. Abidjan, 34 p.

DETIENNE Marcel ; VERNANT Jean-Pierre (1979), La cuisine du sacrifice au pays grec, Paris, Gallimard.

DICK Anthony (2006), Le lavage de cerveau : mythe ou réalité ?, Paris, L'Harmattan, $198 \mathrm{p}$.

DJIBRIL Tamsir Niane (1960), Soundjata ou l'épopée mandingue, Editions Présence africaine, $153 \mathrm{p}$.

DUPIN Henri ; RAIMBAULT Anne.-Marie (1978), Les troubles nutritionnels chez la mère et l'enfant : épidémiologie et prévention. Paris, ENSP, Éditions Saint-Paul.

EZEMBE Ferdinand (2003), L'enfant africain et ses univers, Paris Karthala. FISCHLER Claude (1994), « Magie, charme et aliments », Autrement 149:1019.

FISCHLER Claude (1990), L'omnivore. Le goût, la cuisine et le corps, Paris, Odile Jacob.

FOUGEYROLLAS Patrick (1983), Entre peaux: logis de la différence du handicap à l'autonomie, mémoire de maîtrise, Québec, Université de Laval. 
HAMPATE-BA Amadou (1978), Kaydara, les Nouvelles Editions Africaines, Abidjan- Dakar, 96 p.

HOUIS Maurice (1963), Les noms individuels chez les Mossi, I.F.A.N-Dakar. KOUASSI Kouakou (1997), Des enfants pas comme les autres. Les jumeaux et les autres, in champ psychosomatique, $\mathrm{N}^{\circ} 11 / 12$, pp.95-100.

LEVI-STRAUSS Claude (1962-2), Le totémisme aujourd'hui, Paris, PUF. LEVI-STRAUSS Claude (1962-1), La pensée sauvage, Paris, Agora Pocket , 1990 .

MEMEL-FOTE Harris (1998), Les représentations de la santé et de la maladie chez les Ivoiriens, Paris, L'Harmattan, 209 p.

MORO Marie Rose (1994), Parents en exil. Psychopathologie et migration, Paris PUF.

POULAIN Jean-Pierre (2002), Sociologies de l'alimentation, les mangeurs et l'espace social alimentaire. Paris, PUF, 288 pages.

SCHNAPPER Dominique (2007), Qu'est-ce que l'intégration?, Paris, Gallimard, $240 \mathrm{p}$.

ZEMPLENI Andras ; RABAIN Jamin (1965), l'enfant nit-ku-bu, un tableau psychopathologique traditionnel chez les wolofs et Lébon du Sénégal, nouvelle Revue d'ethnopsychiatrie, psychopathologie africaine, 1,3, 329-441. 